\title{
Synkinetic movements of the eyelid: a case with some unusual mechanisms of paradoxical lid retraction
}

\author{
W. G. BRADLEY AND B. K. TOONE \\ From the Department of Neurology, Regional Neurological Centre, General Hospital, Newcastle upon Tyne
}

\begin{abstract}
A paradoxical or synkinetic movement of an eyelid is the involuntary retraction of the lid associated with other motor activity which normally is functionally separate (Duke-Elder, 1964). An example is the Marcus Gunn phenomenon of retraction of the eyelid on opening the mouth, or protruding the jaw to the opposite side. The eyelid involved in these movements usually shows ptosis at rest. The mechanism of such movements is unknown, and has aroused much speculation. Knowledge of the variety of actions which may be associated with paradoxical lid retraction may clarify the nature of these movements. The present case, in which retraction was associated with activation of the ipsilateral sternomastoid muscle, and with smiling, coughing, and the Valsalva manoeuvre, is reported to add to this knowledge.
\end{abstract}

\section{CASE REPORT}

A woman of 42 years (N.G.H. no. N21938) presented with numbness and weakness of the left leg for a year, and the signs of a spastic paraparesis. All modalities of sensation were subjectively impaired on the left leg below the knee. A myelogram showed a moderate size osteophytic bar at the C3/4 disc space, without hold-up of the flow of contrast medium, and with no other abnormality in the spinal canal. The total protein content of the cerebrospinal fluid was $56 \mathrm{mg} . / 100 \mathrm{ml}$., and of this total $31 \%$ was gamma globulin. There was no pleocytosis. It was felt that the patient was probably suffering from both multiple sclerosis and cervical myelopathy.

From birth she had had a partial left ptosis (Fig. 1), and had noticed that the eyelid would involuntarily retract at times. This retraction, which exposed a large amount of sclera on the left, was most marked on sitting up from the lying position while looking at the feet (Fig. 2). It was also produced by contraction of both sternomastoid muscles together, and by contraction of the left sternomastoid alone. Activation of the right sternomastoid did not produce lid retraction. Involuntary smiling, coughing, and the Valsalva manoeuvre also produced this paradoxical retraction. The phenomenon could be repeatedly elicited but it fatigued slightly. It did not occur with voluntary facial movements, jaw or eye movements, protrusion of the tongue, inspiration, nor on covering the right eye. Eye movements were completely normal in all directions. The left pupil was larger than the right by less than $0.5 \mathrm{~mm}$., and the pupillary reactions were normal. There was no enophthalmos, sweating was normal on both sides of the face, and the cilio-spinal reflex was intact bilaterally.

At different times the following drugs were administered, all in sufficient dosage to produce systemic symptoms: atropine $0.6 \mathrm{mg}$. intramuscularly; carbachol $0.25 \mathrm{mg}$. intramuscularly; adrenaline $1 \mathrm{mg}$. subcutaneously; edrophonium hydrochloride $10 \mathrm{mg}$. intravenously. None had any effect on the resting ptosis, nor on the retraction induced by appropriate manoeuvres. Phentolamine, $5 \mathrm{mg}$. intravenously, also had no effect.

An electromyogram was performed with surface recording electrodes over the left levator anguli oris and over the lower part of the left sternomastoid. There was no evidence of a common innervation of the facial and left sternomastoid muscles, other than minimal activity recorded with the sternomastoid electrodes which was probably related to contraction of the platysma. Retraction of the lid occurred with smiling, coughing, and with the Valsalva manoeuvre in the absence of activity in the sternomastoid and also on turning the head to the right in the absence of activity in the left facial musculature.

\section{DISCUSSION}

This patient had recently developed symptoms and signs which were probably due to both multiple sclerosis and cervical spondylosis, but the fact that the ptosis and paradoxical lid retraction had been present since childhood makes it unlikely that the recent neurological developments were in any way related to the eye condition. Paradoxical lid retraction has been described with eye movements, particularly on looking laterally and downwards. In the Marcus Gunn phenomenon retraction occurs on opening the mouth and protruding the jaw to the opposite side. Synkinesis associated with inspiration (Brain, 1933) and with contraction of the sternomastoid muscles and protrusion of the tongue 


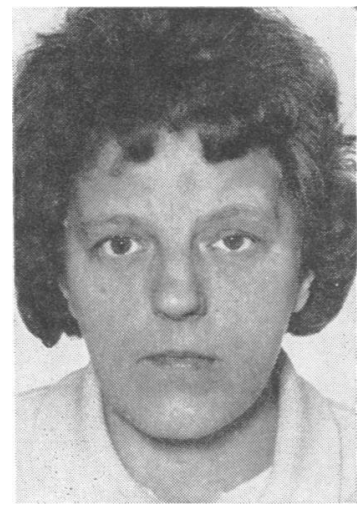

FIG. 1

FIG. 2

FIG. 1. Appearance at rest.

FIG. 2. Appearance on raising the head from the lying position.

(Parry, 1957) in association with the Marcus Gunn phenomenon has also been described. Parry (1957) described and illustrated a case in which there was paradoxical left upper lid retraction associated with right sternomastoid contraction and mentioned that there was a coexistent superior rectus palsy on the same side.

Walsh (1957) described two patients in whom swallowing produced paradoxical retraction of a ptosed right eyelid. In a personal case opening the mouth had no effect, but moving the jaw to the left produced retraction while movement to the right increased the ptosis. During the induction of anaesthesia the lid elevated with each inspiration. $\mathrm{He}$ also described a patient of Drs. Calhoun Jr. and Manchester, in whom swallowing, abduction of the eye, protrusion of the tongue, and contraction of the sternomastoid muscle all produced paradoxical lid retraction, though facial or masticatory movements had no effect. To the best of our knowledge, the present patient is the first to be reported in whom synkinesis occurred with smiling. The fact that retraction occurred with isolated activity in the facial muscles and the sternomastoid muscle indicates that each association constitutes a separate synkinetic mechanism.

The nervous pathways involved in these reflexes are obscure. The fact that smiling produced lid retraction, while voluntary facial movement did not, implicates supranuclear facial pathways. The synkinesis between left steınomastoid and left lid retraction is specific, for retraction was not elicited by activity in the left trapezius muscle, which is also innervated by the left accessory nerve. Synkinesis during coughing and in the Valsalva manoeuvre suggests a possible association in this case between lid retraction and vagus nerve activity.

The existence of these extensive neuro-anatomical associations must make a congenital abnormality of neuronal connexions within the brainstem the most likely explanation of these synkinetic movements.

\section{SUMMARY}

A patient is reported in whom a congenital left ptosis was associated with synkinetic lid retraction on activation of the left sternomastoid muscle, during coughing and with the Valsalva manoeuvre. Smiling, but not voluntary facial movements, also produced paradoxical lid retraction. Retraction could not be elicited by any other volitional movements of muscles in the head and neck. The possible anatomical substrate of this syndrome is discussed.

We are grateful to Dr. J. N. Walton for permission to report this case, and to the Department of Photography of the University of Newcastle upon Tyne for the illustrations.

\section{REFERENCES}

Brain, W. R. (1933). Diseases of the Nervous System, 1st ed. Oxford University Press, London.

Duke-Elder, S. (1964). System of Ophthalmology, Vol. 3, p. 2. Kimpton, London.

Parry, R. (1957). An unusual case of the Marcus Gunn syndrome Trans. Ophthal. Soc. U.K., 77, 181-185.

Walsh, F. B. (1957). Clinical Neuro-Ophthalmology, 2nd ed. Williams and Wilkins, Baltimore. 